\title{
Modelling of the Wireless Propagation Characteristics inside Aircraft
}

\author{
Carl James Debono, Reuben A. Farrugia and Keith Chetcuti \\ University of Malta \\ Malta
}

\section{Introduction}

Advances in wireless communications technology and more sophisticated portable devices have led to a drastic increase in wireless services and applications. This advancement was made possible through hardware improvements which allow more functions to be implemented in smaller sized devices. The demand for more wireless services has pushed the industry and the research community to increase the communication data rates, connectivity, and availability. The increase in the user base has also brought a decrease in the cost of the services.

Although wireless access is becoming available in all public places around the world, this is still not the case for the air passengers who are cut off from wireless services during the duration of the flight. Deployment of wireless technology inside aircraft is still a hot issue due to uncertainties related to interference. Yet, the European Commission has prepared the legal framework for wireless connectivity inside aircraft in April 2008 (Commission, 2008). Aircraft manufacturers will benefit from this technology by exploiting wireless networks to reduce the cable complexity, hence the weight of the aircraft, and by providing new in-flight services, such as online passenger meal selection, service request and video on demand. On the other hand, provisioning of wireless service during flight would profit passengers, since they gain seamless access to common wireless services, such as phone, Internet, and multimedia communications.

A wireless network can always be designed using a measurement campaign, whereby transmitters are placed in strategic locations inside the aircraft and measurements are taken at all points inside the aircraft. This solution is very expensive and requires repetitive readings to measure the propagation losses at all the frequencies lying within the frequency band of interest. These measurements have to be done on all the aircraft models and for every different furniture configuration. While the latter is not much of concern in commercial aircrafts, since these are quite similar in terms of furniture location, this creates a sensible issue in the business jet market, where each aircraft's configuration is tailor-made for the customer. With such requirements, it is not possible to find the ideal antenna locations using measurement techniques.

This calls for the development of an accurate computer model that can estimate the propagation characteristics inside any aircraft. This model must be capable of estimating the power level that can be received at any point inside the enclosed structure, thus creating a 
propagation map. Therefore, any changes in aircraft model and/or its configuration can be easily accommodated and the propagation map recomputed. This map can then be used to determine the parameters associated with the quality of the wireless channel, hence making the characterisation of the wireless channel possible.

In this chapter we present the application of ray tracing techniques, which are based on geometric optics, to model an IEEE 802.11a wireless system propagation map. This technique has become viable with the increased computational power and speeds available in today's computer technology. The developed propagation model can be used to maximise the coverage within the aircraft cabin and to minimise the power emitted from each access point, by determining the optimum location of its antenna. The cabin presents an interesting scenario because the space within it is limited and there is a high density clutter, because of the furniture and seats, resulting in merely any line-of-sight transmission between the server and the client locations. This implies that propagation inside aircraft is mainly due to reflection, transmission and scattering. Also the cabin does not have any attenuation caused by walls as present in normal office environments. We will also discuss how multipath, coherence bandwidth and time dispersion parameters can be obtained, from the computer model, and used to model the channel. These parameters indicate whether inter-symbol and inter-carrier interference will be experienced by the channels.

\section{IEEE 802.11a model}

\subsection{Overview of the IEEE 802.11 standards}

The IEEE 802.11 standards deployed today are a result of technological advancements, both in hardware and software. The first IEEE 802.11 standard was deployed in 1997 with a maximum throughput of $2 \mathrm{Mbps}$ (Crow, B.P. et al, 1997). This has gone a long way and current amendments are looking at high speeds of more than $100 \mathrm{Mbps}$ (Paul, T.K. \& Ogunfunmi, T. 2008). The IEEE 802.11 standard has a mandatory throughput of $1 \mathrm{Mbps}$ which can be extended to 2Mbps. The standard permits three physical layer implementations, namely, frequency hopping spread spectrum (FHSS), direct sequence spread spectrum (DSSS), and Infra red (IR). The FHSS technique uses Gaussian frequency shift keying (GFSK) for modulation, while DSSS and IR utilise differential binary shift keying (DBPSK) / differential quadrature phase shift keying (DQPSK) and pulse position modulation (PPM) respectively (Crow, B.P. et al, 1997).

IEEE 802.11a presents data rates of up to $54 \mathrm{Mbps}$ and uses the $5 \mathrm{GHz}$ unlicensed national information infrastructure (U-NII) frequency band. The physical layer uses orthogonal frequency division multiplexing (OFDM) multicarrier transmission. The modulation schemes used are binary phase shift keying (BPSK), quadrature phase shift keying (QPSK), and quadrature amplitude modulation (QAM) (IEEE standard, 2003).

\subsection{Orthogonal frequency division multiplexing}

The application of OFDM to wireless communications has gained ground in the last decade. The main features offered by this technology are: (i) OFDM is spectrally efficient (Can De Beek, J.J. et al, 2002) and (ii) it increases robustness against frequency selective fading (Yomo, H. et al, 2005). The latter is because in a multi-carrier system, it is highly improbable that a fade will affect all the subcarriers and the system can still function. 
The orthogonal subcarriers overlap one another making the system bandwidth efficient when compared to non-overlapping multi-carrier systems. This characteristic makes sure that the subcarriers do not interfere with each other. The input stream is divided into $N$ data streams, corresponding to the number of subcarriers, and each subcarrier modulates one of these low rate data streams. A multiplex of these modulated subcarriers results in an OFDM symbol.

One of the problems with OFDM is that orthogonality can be lost if the signal is transmitted over a dispersive channel causing inter-carrier interference (ICI). This problem can be solved by introducing a cyclic prefix (Peled, A. \& Ruiz, A., 1980). This solution also prevents intersymbol interference (ISI) between consecutive OFDM symbols. This means that simple onetap equalizers, implemented at the receiver, are enough to offer protection against ICI and ISI, making the use of OFDM possible in wireless environments.

\subsection{IEEE 802.11a PHY layer}

The physical (PHY) layer interfaces the medium access control (MAC) layer to the wireless medium. The table below (IEEE standard, 2003) gives a summary of the permissible data rates and their characteristics. A block diagram illustrating how a symbol is generated in the PHY layer is presented in Figure 1.

\begin{tabular}{|c|c|c|c|c|c|c|}
\hline Mode & $\begin{array}{c}\text { Data rate } \\
(\mathrm{Mbps})\end{array}$ & Modulation & $\begin{array}{c}\text { Coding rate } \\
(\mathrm{R})\end{array}$ & $\begin{array}{c}\text { Coded bits } \\
\text { per } \\
\text { subcarrier }\end{array}$ & $\begin{array}{c}\text { Coded bits } \\
\text { per OFDM } \\
\text { symbol }\end{array}$ & $\begin{array}{c}\text { Data bits per } \\
\text { OFDM } \\
\text { symbol }\end{array}$ \\
\hline 1 & 6 & BPSK & $1 / 2$ & 1 & 48 & 24 \\
\hline 2 & 9 & BPSK & $3 / 4$ & 1 & 48 & 36 \\
\hline 3 & 12 & QPSK & $1 / 2$ & 2 & 96 & 48 \\
\hline 4 & 18 & QPSK & $3 / 4$ & 2 & 96 & 72 \\
\hline 5 & 24 & 16QAM & $1 / 2$ & 4 & 192 & 96 \\
\hline 6 & 36 & 16QAM & $3 / 4$ & 4 & 192 & 144 \\
\hline 7 & 48 & 64QAM & $2 / 3$ & 6 & 288 & 192 \\
\hline 8 & 54 & 64QAM & $3 / 4$ & 6 & 288 & 216 \\
\hline
\end{tabular}

Table 1. IEEE 802.11a physical layer parameters.

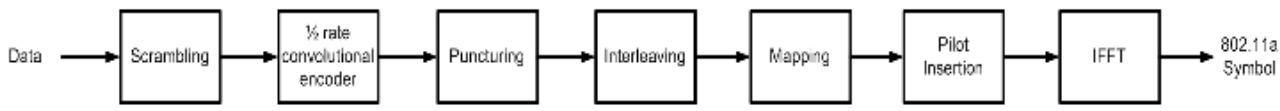

Fig. 1. Block diagram of IEEE 802.11a symbol generation

The data coming from the higher layers is first scrambled and protected by the convolutional encoder. Puncturing is applied to these symbols, when necessary, to obtain the output symbol. Symbol interleaving is applied to protect the sequence from burst errors, by introducing sparsity between consecutive data elements. The new data sequence is mapped using the operating modulating scheme and a serial-to-parallel converter divides the data stream into 48 different streams, corresponding to the number of carriers. Pilot signals are then inserted to aid in the demodulation and the channel estimation process at the receiver side. The inverse Fourier transform (IFFT) converts the signal back into the time 
domain. A cyclic prefix is added to each symbol before re-converting the streams to a single data stream. This cyclic prefix helps in preventing ISI between adjacent symbols and ICI between adjacent carriers. The length of this prefix is a design parameter which depends on the application, since it must be larger than the delay spread of the channel to guarantee that no ISI or ICI distortion effects are introduced in the system.

The OFDM PHY layer divides the information signal across 52 subcarrier frequencies. From these, 48 are used for data transmission and 4 are used by the pilot signals. Another 12 subcarriers are used as cyclic prefix. The available bandwidth is $20 \mathrm{MHz}$ and each subcarrier is separated by $312.5 \mathrm{kHz}$. With this configuration, each symbol has a duration of $4 \mu \mathrm{s}$, where the IFFT information has a period of $3.25 \mu$ s while the cyclic prefix uses the remaining $0.75 \mu$ s.

\section{Propagation model}

The geometry inside an aircraft contains high density clutter, very different from typical office and home scenarios. This means that severe wireless propagation conditions prevail. The advances in computer computation power has made ray tracing modelling possible. Ray tracing techniques offer an adequate representation of the wireless propagation characteristics inside closed, high clutter environments (Diaz, N.R. \& Achilli, C., 2003).

The method uses the theory of geometric optics (GO) and is a brute-force solution. Rays are launched from a transmitter at a fixed power level and an estimate of the power levels along the path is found. These rays will undergo reflection, refraction and diffraction as they impinge on the structure of the cabin and the furniture found along the path. The result is a three-dimensional map of the power levels inside the aircraft. This map therefore represents an estimate of the field strength that would be received by mobile terminals at any position inside the aircraft.

\subsection{Ray tracing technique}

The two most popular ray tracing techniques are: (i) the method of images, and (ii) the method of shoot and bounce. The first solution gives the exact radiation pattern when the environment is smooth, infinite, or made up of semi-infinite perfect electrically conducting surfaces arranged in a limited set of canonical geometries (Diaz, N.R. \& Achilli, C., 2003). The second method is more suitable for complex environments and is therefore used for the propagation modelling inside the aircraft.

In the cabin environment, the signal wavelength, i.e. $5.7 \mathrm{cms}$, is much smaller than the dimensions of the clutter inside the structure and therefore the approximation of the GO hypothesis holds. This implies that ray tracing can be safely used to model the system. A ray is associated with a local plane wave which can be represented by (Diaz, N.R. \& Achilli, C., 2003):

$$
\nabla^{2} \psi+k^{2} n^{2} \psi=0
$$

where $\psi$ is the waveform function which governs the scalar wave propagation, $k$ is the wave number, and $n$ is the reflection index of the medium. Solving for $\psi$ gives (Diaz, N.R. \& Achilli, C., 2003):

$$
\psi=A e^{-j k S}
$$


where $A$ is a function which determines the amplitude of the wave and the function $S$ determines the direction and phase. Using equations (1) and (2), we can conclude that if

$$
\frac{\nabla^{2}}{k^{2}}<<n^{2}
$$

a solution which does not depend on the frequency of operation can be obtained and therefore geometric optics can be used.

As the rays pass through the medium they will experience reflection, refraction and diffraction. This occurs because of the obstacles found in the path between the transmitters and the receivers. If we assume that the obstacles found in the cabin environment are made up of homogeneous material, we simplify the model as each surface can now be described through its dielectric constant, magnetic permittivity and conductivity. This assumption implies that diffraction is not taken into consideration.

If two media possessing different conductivity and permittivity characteristics are assumed to be separated by an infinite plane, then we can obtain equations that relate the reflected electromagnetic wave to the incident wave and the properties of the media. Polarization effects must also be considered and this is done by splitting the electric field into two components; a component which lies parallel to the incident surface and another one which lies perpendicular to it. The refracted and reflected rays can be estimated by multiplying each component to the corresponding Fresnel's coefficient, given by (James, G.L., 1986):

$$
\begin{gathered}
R_{\perp}=\frac{\cos \theta-\sqrt{\hat{\varepsilon}_{r}-\sin ^{2} \theta}}{\cos \theta+\sqrt{\hat{\varepsilon}_{r}-\sin ^{2} \theta}} \\
R_{\|}=\frac{\hat{\varepsilon}_{r} \cos \theta-\sqrt{\hat{\varepsilon}_{r}-\sin ^{2} \theta}}{\hat{\varepsilon}_{r} \cos \theta+\sqrt{\hat{\varepsilon}_{r}-\sin ^{2} \theta}} \\
T_{\perp}=\frac{2 \cos \theta}{\hat{\varepsilon}_{r} \cos \theta+\sqrt{\hat{\varepsilon}_{r}-\sin ^{2} \theta}} \\
T_{\|}=\frac{2 \sqrt{\hat{\varepsilon}_{r}} \sin \theta}{\hat{\varepsilon}_{r} \cos \theta+\sqrt{\hat{\varepsilon}_{r}-\sin ^{2} \theta}}
\end{gathered}
$$

where $\theta$ is the angle of incidence, and $\hat{\varepsilon}_{r}=\varepsilon_{r}-j(\eta / 2 \pi) \sigma \lambda$ is the relative complex dielectric constant. In this equation, $\varepsilon_{r}$ is the relative dielectric constant, $\sigma$ is the conductivity of the materials, $\lambda$ is the wavelength in meters, $R$ is the reflection coefficients, and $T$ is the refraction coefficients. These relations are valid in this case as the medium is air, which presents a dielectric constant of $\varepsilon_{0}$ (Diaz, N.R. \& Achilli, C., 2003).

The Fresnel reflection coefficients will account for reflections coming from smooth surfaces. However, this is not the case for the environment being considered. The Rayleigh criterion (Bothias, L., 1987) can be used as a roughness test. When a surface is rough the incident's 
ray energy will be diffused in angles other than the main angle of reflection, and therefore there is a reduction in the energy of the main reflected ray (Landron, O. et al, 1993). The critical height, $h_{c}$, in meters, defined by the Rayleigh criterion is given by:

$$
h_{c}=\frac{\lambda}{8 \cos \theta_{i}}
$$

where $\lambda$ is the wavelength of the signal and $\theta_{i}$ is the angle of incidence.

A surface is considered to be rough if the protuberances exceed $h_{c}$. In such cases the reflection coefficients $\left(R_{\perp}\right.$ and $\left.R_{\|}\right)$have to be modified by the scattering loss factor (Hashemi, H., 1993):

$$
\rho_{s}=\exp \left[-8\left(\frac{\pi \sigma_{h} \cos \theta_{i}}{\lambda}\right)^{2}\right] I_{o}\left[8\left(\frac{\pi \sigma_{h} \cos \theta_{i}}{\lambda}\right)^{2}\right]
$$

where $\sigma_{h}$ is the standard deviation of the surface height. The reflection coefficients thus become:

$$
\begin{gathered}
\left(R_{\perp}\right)_{\text {rough }}=\rho_{s}\left(R_{\perp}\right)_{\text {smooth }} \\
\left(R_{\|}\right)_{\text {rough }}=\rho_{S}\left(R_{\|}\right)_{\text {smooth }}
\end{gathered}
$$

Within the IEEE 802.11a frequency band, the critical height varies from $7 \mathrm{~mm}$ at an angle of $1^{\circ}$ to $40.9 \mathrm{~cm}$ at an angle of $89^{\circ}$. Inside the cabin, we will not find large roughness in the materials used. Figure 2 shows the profile of the scattering roughness factor for an IEEE 802.11a system. From this figure, it can be seen that the ray can loose up to $75 \%$ of its energy when it hits a surface.



Fig. 2. Scattering roughness factor against incident angle at $5.25 \mathrm{GHz}$ 


\subsection{Signal strength propagation model}

Considering the layout of a typical A340-600 cabin, an aircraft model which includes the furniture is developed. The signal strength at each location inside the aircraft is determined by placing the access points at fixed locations inside the aircraft. Rays are then launched from each transmitting antenna. By vectorially adding all the rays passing through all the points inside the cabin, we obtain an estimate of the signal strength at each position within the aircraft. Therefore, a propagation map which indicates the radio coverage is created.

A ray leaving the transmitter will travel in free space until it impinges on a surface. At this point it is reflected or reflected and refracted as illustrated in figure 3 . The rays that result from this interaction are launched again with the new power level from the point of collision. This process will be repeated until the power of the ray falls below a pre-determined threshold.

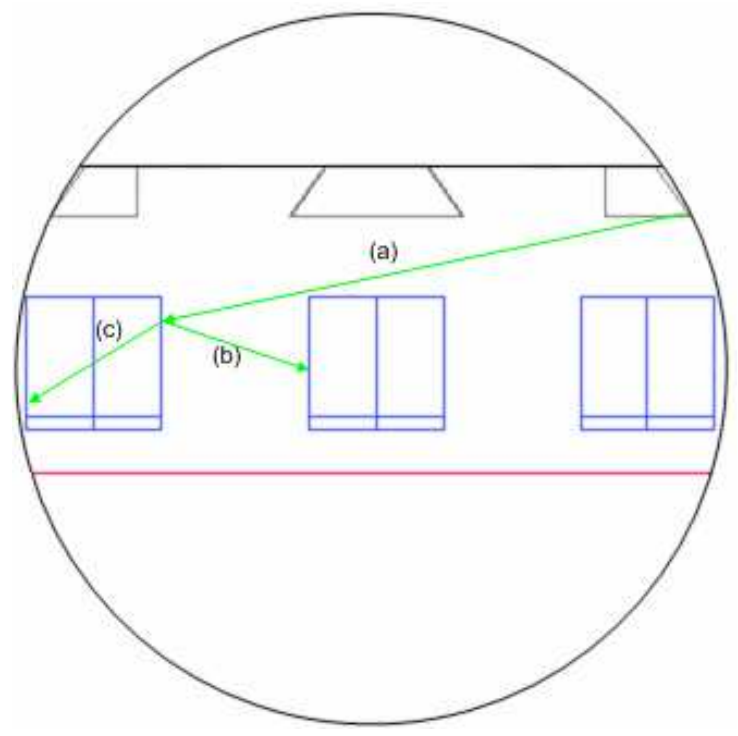

Fig. 3. The ray tracing technique (a) main ray, (b) reflected ray, and (c) refracted ray

The power that is received by the path of the $\mathrm{k}^{\text {th }}$ ray that reaches a single point, is given by (Diaz, N.R. \& Achilli, C., 2003):

$$
P_{k}=P_{T}\left(\frac{\lambda}{4 \pi r}\right)^{2} G_{T} G_{R} \prod_{i} R_{i} \prod_{j} T_{j}
$$

where $P_{T}$ is the transmit power in Watts, $G_{T}$ and $G_{R}$ are the transmitter and receiver gains respectively, $\lambda$ is the wavelength in meters, $r$ is the total unfolded path length in meters, $R_{i}$ and $T_{j}$ are the reflection and refraction coefficients respectively (determined by equations (4) to (7)), and $i$ and $j$ are the indexes that increment over reflection and refraction respectively. The polarization model can be simplified using techniques found in (Chizhik, D. et al., 1998). The phase, $\phi_{k}$, of the received field is computed from the fast fading prediction, where $\phi_{k}$ is a function of the unfolded path length and the number of reflections. The signal strength at a point in the aircraft can thus be evaluated using: 


$$
P_{R}=\left|\sum_{k} \sqrt{P_{k}} e^{-j \phi_{k}}\right|^{2}
$$

and

$$
\phi_{k}=k r+R_{s h}
$$

where $k$ is the wave number per meter, $r$ is the length of the path in meters, and $R_{s h}$ is the phase shift due to the reflection in radians.

\subsection{The reflection model}

Reflection is implemented according to Fermats principle. The direction of the reflected ray is found using:

$$
\vec{r}=\vec{v}-2(\vec{n} \cdot \vec{v}) \vec{n}
$$

where $\vec{v}$ is the incident ray, $\vec{n}$ is the normal to the plane of incidence, and - is the dot product operator. In the cabin environment we will experience a large number of reflections, from every surface. Using Fresnels coefficients, defined above, the rays will experience a phase shift of $\pi$ radians every time there is a reflection. The field power of the reflected signal becomes:

$$
P_{r}=\sqrt{P_{r \|}^{2}+P_{r \perp}^{2}}
$$

where

$$
\begin{gathered}
P_{r \|}=P_{i \|} \cdot\left|R_{\|}\right|^{2} \\
P_{r \perp}=P_{i \perp} \cdot\left|R_{\perp}\right|^{2} \\
P_{i \|}=P_{i} \cos \theta
\end{gathered}
$$

and

$$
P_{i \perp}=P_{i} \sin \theta
$$

The subscripts $r$ and $i$ represent the reflected ray and the incident ray respectively.

The GO principle can also be used to model the propagation of the waves as they hit the curved walls of the aircraft. This can be done because the radius of curvature of this surface is very large compared to the wavelength of the signal. Therefore, the incident ray is reflected at the tangential plane of the surface at the point where the incident ray impinges on the cabin wall.

\subsection{The refraction model}

The propagating signal experiences refraction whenever the ray hits an obstacle. The rays which are refracted in a direction of travel which lies outside the aircraft are assumed to be 
absorbed within the material. This is because we are not interested in the rays which leave the aircraft. The direction of the refracted ray can be calculated using (Diaz, N.R. \& Achilli, C., 2003):

$$
\vec{t}=n \vec{v}-\left(n(\vec{v}, \vec{n})+\sqrt{1-n^{2}\left(1-(\vec{v}, \vec{n})^{2}\right)}\right) \vec{n}
$$

where $n=n_{1} / n_{2}$ and $n_{1}$ and $n_{2}$ are the refraction indexes of the two different media.

The refracted power is calculated using the following:

$$
P_{t}=\sqrt{P_{t \|}^{2}+P_{t \perp}^{2}}
$$

where

$$
\begin{gathered}
P_{t \|}=P_{i \|} \cdot\left|T_{\|}\right|^{2} \\
P_{t \perp}=P_{i \perp} \cdot\left|T_{\perp}\right|^{2}
\end{gathered}
$$

where the subscripts $i$ and $t$ represent the incident ray and refracted ray respectively. The model assumes that the obstacles encountered by the rays have constant dielectric properties.

\subsection{The access point model}

Each IEEE 802.11a access point is assumed to have an omni-directional antenna. This simplifies things as the access point can be modelled as a point source which radiates the rays uniformly in the three-dimensional space. The Monte Carlo stochastic launching model (Diaz, N.R. \& Achilli, C., 2003) is then used to model each transmitter deployed on the aircraft. This will generate rays having random directions within the cabin with equal probability. This ensures that no region within the cabin will contain more rays than another, something which would otherwise skew the results.

The one-dimensional probability density functions are given by (Diaz, N.R. \& Achilli, C., 2003):

$$
\begin{gathered}
p_{\theta}(\theta)=\int_{0}^{2 \pi} p_{\theta, \phi}(\theta, \phi) d \phi=\frac{\sin \theta}{2} \\
p_{\phi}(\theta)=\int_{0}^{\pi} p_{\theta, \phi}(\theta, \phi) d \theta=\frac{1}{2 \pi}
\end{gathered}
$$

where $\phi$ and $\theta$ are the spherical coordinates, with $0 \leq \phi \leq 2 \pi$ and $0 \leq \theta \leq \pi$. These two randomly distributed variables are generated using:

$$
\theta=\arccos \left(1-2 \xi_{1}\right)
$$

and 


$$
\phi=2 \pi \xi_{2}
$$

where $\xi_{1}$ and $\xi_{2}$ are random variables which are distributed in $[0,1]$ and in $[0,1)$ respectively.

\subsection{Multipath characteristics}

We know that the aircraft layout has a very high object density. These objects produce a lot of reflections and refractions as the signals propagate. Therefore, the signal strength arriving at a receiver is highly affected by the large number of multipath signals arriving at that location. These signals will be added vectorially by the receiver hardware. The impulse response can be used to obtain the channel characteristics in these scenarios.

The impulse response of the channel can be modelled using (Hashemi, H., 1993) and (Saleh, A.A.M. \& Valenzuela, R.A., 1987). The time invariant impulse response is expressed as a sum of $k=1 \ldots N$ multipath components, each having a random amplitude $a_{k}$, delay $\tau_{k}$, and phase $\theta_{k}$ :

$$
h(t)=\sum_{k=1}^{N} a_{k} \partial\left(t-\tau_{k}\right) e^{j \theta_{k}}
$$

The three main distributions that are used in communications theory to model multipath effects are the (i) Nakagami, (ii) Rician, and (iii) Rayleigh distributions. At a point inside the aircraft, the signal will experience different fading characteristics as the number of multipath components reaching that point varies. The Rician distribution is more appropriate to scenarios having a dominant line-of-sight signal, which is not the case for the cabin environment. The choice is therefore between the Nakagami and the Rayleigh distribution models. The study in (Can De Beek, J.J. et al, 2002) shows that the multipath distribution of an indoor channel can be better represented by a Nakagami distribution. This distribution is characterised by the cumulative density function:

$$
p(x, \mu, \omega)=\frac{2 \mu^{\mu}}{\Gamma(\mu) \omega^{u}} x^{2 \mu-1} \exp \left(-\frac{\mu}{\omega} x^{2}\right)
$$

where $\mu$ is a shape parameter and $\omega$ controls the spread of the distribution.

These distribution parameters have to be extracted from the simulation model. In order to do this, the total number of multipath rays and the maximum and minimum delay times must be recorded for each location inside the cabin. The area inside the cabin is divided into areas, called cells. All the data that is located within the same cell number, which represents the cell distance from the transmitter, is clustered together. The Nakagami model is then fit to this data. Hence, this will give a list of fit parameters that model the multipath propagation inside the cabin.

\subsection{Time dispersion parameters}

The IEEE 802.11a channel parameters are characterised by the time dispersion parameters. The main components are composed of: (i) the mean excess delay, and (ii) the root-meansquare (rms) delay spread. These parameters give an estimate of the expected performance that the wireless system will achieve if deployed in the cabin environment. These parameters are then input to the top level IEEE 802.11a system model to obtain the bit error 
rate (BER) of the channel. The BER results are then used to get an estimate of the quality of service (QoS) and other data transmission characteristics.

The mean excess delay, $\tau_{m}$, is defined as:

$$
\tau_{m}=\frac{\sum_{k}\left\{\left(\tau_{k}-\tau_{1}\right) a_{k}^{2}\right\}}{\sum_{k} a_{k}^{2}}
$$

while the rms delay spread, $\tau_{r m s}$, is:

$$
\tau_{r m s}=\left(\frac{\sum_{k}\left\{\left(\tau_{k}-\tau_{m}-\tau_{1}\right)^{2} a_{k}^{2}\right\}}{\sum_{k} a_{k}^{2}}\right)^{\frac{1}{2}}
$$

where $\tau_{k}$ is the delay of the $\mathrm{k}^{\text {th }}$ multipath ray with a normalised amplitude of $a_{k}$, and $\tau_{1}$ is the delay of the line-of-sight signal.

\subsection{Coherence bandwidth}

The coherence bandwidth, $B_{c}$, is a measure of the range of frequencies over which two frequency components are likely to have amplitude correlation (Rappaport, T.S., 2002). This bandwidth is related to the rms delay spread. Two signals centered at frequencies that have a separation which is less than or equal to $B_{c}$ will have similar channel impairments. Otherwise the signals can experience frequency selective fading.

For a frequency correlation function of 0.9 or above, $B_{c}$ can be approximated by (Lee, W.C.Y., 1989):

$$
B_{c} \approx \frac{1}{50 \tau_{r m s}}
$$

while for a frequency correlation function above 0.5 , this approximation becomes:

$$
B_{c} \approx \frac{1}{5 \tau_{r m s}}
$$

Results within a business jet can be found in (Debono, C.J. et al, 2009). For the system to guarantee that the receiver does not experience inter-symbol interference and/or interchannel interference, the guard interval at any location within the cabin must be less than 800ns (as specified in the IEEE 802.11a standard). Moreover, the system will only introduce flat fading if the coherence bandwidth is greater than the bandwidth of the subcarriers, which is equal to $312.5 \mathrm{kHz}$.

\section{Simulation results}

\subsection{The cabin model}

A three-dimensional model of the cabin can be developed using any computer aided design (CAD) software. This model can then be imported in the simulation software, which for this 
work was developed in Matlab $^{\circledR}$. The propagation characteristics presented here relate to an Airbus A340-600 but further results on a Dassault Aviation business jet can be found in (Debono, C.J. et al, 2009) and (Chetcuti, K. et al, 2009). The structure of the aircraft is modelled through a cylinder which represents the fuselage and a horizontal plane to model the floor. Furniture and the stowage bins were also included as shown in Figure 4.

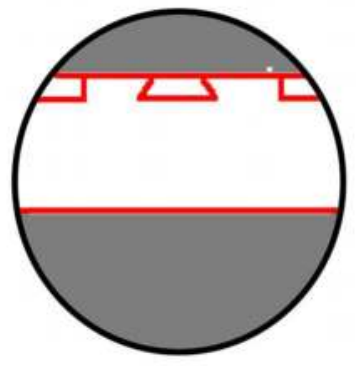

(a)



(b)

Fig. 4. Cross-section of cabin without seats (a), and with seats (b).

The seats have a specific thickness and are modelled as two intersecting planes. The dielectric constant, permittivity and conductivity depend on the material used. Typical values of the materials used inside the cabin of the aircraft are given in Table 2.

\begin{tabular}{|c|c|c|}
\hline Material & $\begin{array}{c}\text { Electric } \\
\text { Conductivity }\end{array}$ & $\begin{array}{c}\text { Relative } \\
\text { Permittivity }\end{array}$ \\
\hline Aluminium & $4 \mathrm{E} 7$ & Inf \\
\hline Leather & $1 \mathrm{E}-2$ & 3 \\
\hline Wood & $1 \mathrm{E}-2$ & 3 \\
\hline
\end{tabular}

Table 2. Electrical characteristics of materials used inside a cabin

\subsection{Simulation environment}

The ray tracing algorithm is implemented in Matlab ${ }^{\circledR}$. A flow chart of the main algorithm is shown in Figure 5. The geometry file used by the developed simulator represents the typical environment of the aircraft under test. The signal strength propagation map is determined by launching 200,000 rays from each transmitter antenna. The equivalent isotropic radiated power from each access point is $30 \mathrm{dBm}$. At any particular cell inside the cabin, the signal strength is determined by summing the power levels of all the rays passing through that point. This implies that the received signal is a distorted version of the transmitted signal.

As discussed in section 3.5, the starting direction of each ray is determined using Monte Carlo techniques, where two random numbers, representing the angles in spherical coordinates, in the range 0 to $2 \pi$ and 0 to $\pi$ respectively are generated. Each ray is traced one cell size at a time, where at each cell position, the simulator assesses whether the ray is still inside the aircraft. If it is found to lie outside the aircraft, then the trace ends there and the 




Fig. 5. Flowchart of the ray tracing method

simulator goes back to the antenna to start a new ray trace. If the ray is still inside the aircraft, the propagation loss is calculated. The new power level is compared to the predetermined threshold, which in our case is set to $-120 \mathrm{dBm}$, and if it is above this threshold a check is performed to test whether the ray has impinged on a surface. The $-120 \mathrm{dBm}$ level is well below the minimum detectable signal for IEEE 802.11a, but because of the multipath effects some margin is required to allow for the eventuality that the vectorially summed power level could still exceed the $-100 \mathrm{dBm}$ limit defined in the standard. The received signal strength at the receiver affects the signal-to-noise ratio (SNR) posing a limit on the maximum useable data rate for error free communication.

\subsection{Results}

Placing just one access point inside the aircraft limits the number of users that can access the network. This occurs because of the limited capacity that would be offered and the radio propagation coverage that can be obtained with reasonable transmit power levels. The higher the power emanating from the access point, the more interference it is likely to cause to the aircraft's electronics. The simulator developed can be used to determine the optimum number of access points and their position within the aircraft. An analysis for the optimum 
antenna locations for a Universal Mobile Terrestrial System (UMTS) is found in (Debono, C.J. \& Farrugia, R.A., 2008).

The resulting propagation map for the A340-600, using four IEEE 802.11a access points, is shown in figures 6 to 9 . Figure 6 presents the view from the antenna plane, Figure 7 shows the top view at the middle of the aircraft, Figure 8 shows the side view, while Figure 9 shows cross-sections looking from the front of the aircraft.



Fig. 6. Propagation map at the antenna plane. The four access points are shown by the areas of maximum signal concentration.

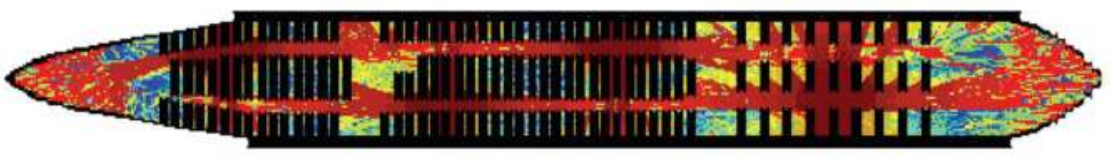

Fig. 7. Propagation map at the middle of the aircraft as seen from the top. 


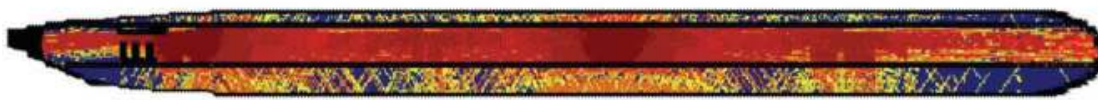

(a)



Fig. 8. The propagation map as seen from the side; (a) aisle, and (b) across a column of seats.



(a)
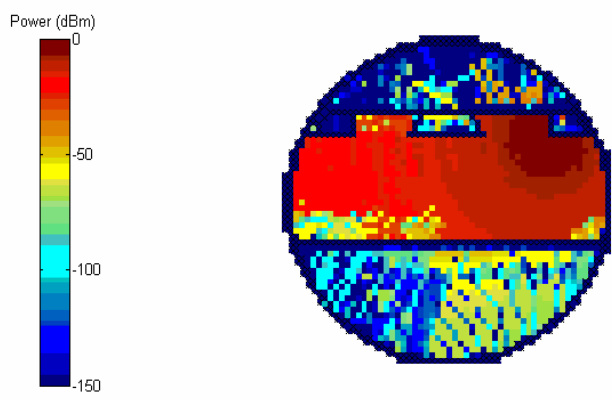

(b)

Fig. 9. The propagation map as seen from the aisle; (a) at the front row, and (b) near one of the access points. 
The simulator has also been used to model the propagation inside a business jet. A measurement campaign was done in this case to compare the results obtained and determine the confidence level of the simulations. The results have been presented in (Chetcuti, K. et al, 2009) and show that the model is reasonably accurate, especially within the cabin area.

\section{Conclusion}

This chapter has given the theoretical background necessary to develop a radio propagation map for an IEEE 802.11a system. The method is based on ray tracing techniques which rely on the theory of geometric optics. This solution can be applied to both commercial aircraft, like the A340-600 used in this case, and business jets. The flexibility of the simulator allows easy modifications to simulate different frequency bands and different furniture location and material. This makes the simulator very attractive especially in the business jet environment, where the interior furniture of each aircraft is specifically designed for each customer.

The simulator allows the user to insert the number of access points required and their location. Using an intelligent optimisation technique, such as neural networks, genetic algorithms and support vector machines, one can find the optimum number of access points and their optimum location within the aircraft. This can be done given some constrains imposed by the wiring system of the aircraft.

Moreover, the propagation map gives an idea of the electromagnetic radiation field strength hitting the fuselage of the aircraft. A similar method can be used for each portable device held by each passenger in the aircraft to simulate the uplink. Therefore, the designer can estimate the electromagnetic interference that is generated by the system. Through optimum design of the system the electromagnetic interference can be kept within acceptable limits and thus ensure that no interference occurs with the aircraft's navigation and control system.

\section{Acknowledgements}

The authors would like to thank Mr. Serge Bruillot from Dassault Aviation for providing us with the model file of their Falcon $\mathrm{X} 7$ business jet and for the measurement campaign referenced in the text.

This work forms a small part of the project E-Cab which is financially supported under the European Union 6th Framework Programme (FP6) (E-Cab Website, 2008). The E-Cab consortium is made up of 30 partners from 13 countries across Europe. The authors are solely responsible for the contents of the chapter which does not represent the opinion of the European Commission.

\section{References}

Bothias, L. (1987), Radio Wave Propagation, McGraw Hill Inc., New York, USA.

Can De Beek, J.J., Odling, P., Wilson, and S.K., Bojesson, P.O. (2002), Orthogonal FrequencyDivision Multiplexing, International Union of Radio Science, 2002. 
Chetcuti, K., Debono, C.J., Farrugia, R.A., and Bruillot, S. (2009), Wireless Propagation Modelling Inside a Business Jet, Proceedings of Eurocon 2009, May 2009, pp. 16401645.

Chizhik, D., Ling, J., and Valenzuela, R.A. (1998), The Effect of Electric Field Polarization on Indoor Propagation, IEEE 1998 International Conference on Universal Personal Communications, October 1998, pp. 459-462.

Commission Decision of [...] on harmonised conditions of spectrum use for the operation of mobile communication services on aircraft (MCA services) in the Community Commision of the European Communities, April 2008.

Crow, B.P., Widjaja, I., Kim, J.G., and Sakai, P.T. (1997), IEEE 802.11 Wireless Local Area Networks, IEEE Communications Magazine, September 1997, pp. 116-126.

Debono, C.J., and Farrugia, R.A. (2008), Optimization of the UMTS Network Radio Coverage On-board an Aircraft, Proceedings of the 2008 IEEE Aerospace Conference, March 2008.

Debono, C.J., Chetcuti, K. and Bruillot, S. (2009), 802.11a Channel Parameters Characterization on board a Business Jet, Proceedings of the 2009 IEEE Aerospace Conference, March 2009.

Diaz, N.R., and Achilli, C. (2003), Cabin Channel Characterization for Personal Communications via Satellite, Proceedings of the 21 $1^{\text {st }}$ International Communications Satellite Systems Conference and Exhibit, 2003.

E-Cab Consortium Website, Online: http:/ / www.e-cab.org

Hashemi, H. (1993), The Indoor Radio Propagation Channel, Proceedings IEEE, vol.81, July 1993.

IEEE Std 802.11a-1999(R2003), Part 11: Wireless LAN Medium Access Control (MAC) and Physical Layer (PHY) specifications High-speed Physical Layer in the $5 \mathrm{GHz}$ Band, 2003.

James, G.L. (1986), Geometric Theory of Diffraction for Electromagnetic Waves, Peter Peregrinus Ltd., London, UK.

Landron, O., Feuerstein, M.J., and Rappaport, S. (1993), In Situ Microwave Reflection Coefficient Measurements for Smooth and Rough Exterior Wall Surfaces, Proceedings of the IEEE 43rd Vehicular Technology Conference, May 1993, pp. 77-80.

Lee, W.C.Y. (1989), Mobile Cellular Telecommunications Systems, McGraw Hill Publications, New York, USA.

Paul, T.K., and Ogunfunmi, T. (2008), Wireless LAN Comes of Age: Understanding the IEEE 802.11n Amendment, IEEE Circuits and Systems Magazine, First Quarter 2008, vol. 8, no. 1 , pp $28-54$.

Peled, A., and Ruiz, A. (1980), Frequency Domain Data Transmission using Reduced Computational Complexity Algorithms, Proceedings of the IEEE International Conference on Acoustics, Speech and Signal Processing, 1980, pp. 964-967.

Rappaport, T.S. (2002), Wireless Communications Principles and Practice, Prentice Hall, New Jersey, USA.

Saleh, A.A.M., and Valenzuela, R.A. (1987), A Statistical Model for Indoor Multipath Propagation, IEEE Journal on Selected Areas Communications, February 1987, pp. 128137. 
Yomo, H., Nguyen, C.H., Kyritsi, P., Nguyen, T.D., Chakraborty, S.S., and Prasad, R., PHY and MAC Performance Evaluation of IEEE 802.11a WLAN over Fading Channels, Institution of Electronics and Telecommunications Engineers (IETE), vol. 51, no. 1, January 2005, pp. 83-94. 


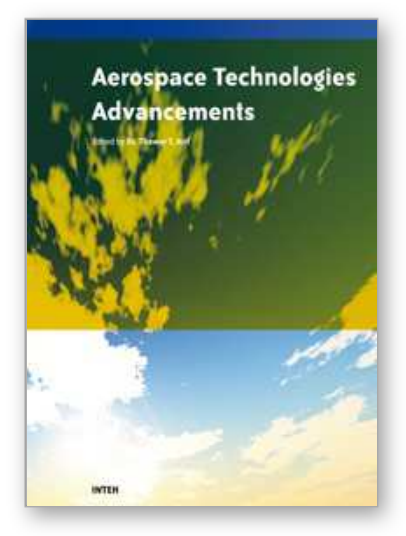

\author{
Aerospace Technologies Advancements \\ Edited by Thawar T. Arif
}

ISBN 978-953-7619-96-1

Hard cover, 492 pages

Publisher InTech

Published online 01, January, 2010

Published in print edition January, 2010

Space technology has become increasingly important after the great development and rapid progress in information and communication technology as well as the technology of space exploration. This book deals with the latest and most prominent research in space technology. The first part of the book (first six chapters) deals with the algorithms and software used in information processing, communications and control of spacecrafts. The second part (chapters 7 to 10) deals with the latest research on the space structures. The third part (chapters 11 to 14 ) deals with some of the latest applications in space. The fourth part (chapters 15 and 16) deals with small satellite technologies. The fifth part (chapters 17 to 20) deals with some of the latest applications in the field of aircrafts. The sixth part (chapters 21 to 25) outlines some recent research efforts in different subjects.

\title{
How to reference
}

In order to correctly reference this scholarly work, feel free to copy and paste the following:

Carl James Debono, Reuben A. Farrugia and Keith Chetcuti (2010). Modelling of the Wireless Propagation Characteristics inside Aircraft, Aerospace Technologies Advancements, Thawar T. Arif (Ed.), ISBN: 978-9537619-96-1, InTech, Available from: http://www.intechopen.com/books/aerospace-technologiesadvancements/modelling-of-the-wireless-propagation-characteristics-inside-aircraft

\section{INTECH}

open science | open minds

\author{
InTech Europe \\ University Campus STeP Ri \\ Slavka Krautzeka 83/A \\ 51000 Rijeka, Croatia \\ Phone: +385 (51) 770447 \\ Fax: +385 (51) 686166 \\ www.intechopen.com
}

\author{
InTech China \\ Unit 405, Office Block, Hotel Equatorial Shanghai \\ No.65, Yan An Road (West), Shanghai, 200040, China \\ 中国上海市延安西路65号上海国际贵都大饭店办公楼 405 单元 \\ Phone: +86-21-62489820 \\ Fax: +86-21-62489821
}


(C) 2010 The Author(s). Licensee IntechOpen. This chapter is distributed under the terms of the Creative Commons Attribution-NonCommercialShareAlike-3.0 License, which permits use, distribution and reproduction for non-commercial purposes, provided the original is properly cited and derivative works building on this content are distributed under the same license. 